\title{
PLANE PROJECTIONS OF A SMOOTH SPACE CURVE
}

\author{
TRYGVE JOHNSEN \\ Department of Mathematics, University of Bergen \\ Allégt. 55, N-5007 Bergen, Norway \\ E-mail: Trygve.Johnsen@mi.uib.no
}

\begin{abstract}
Let $C$ be a smooth non-degenerate integral curve of degree $d$ and genus $g$ in $\mathbb{P}^{3}$ over an algebraically closed field of characteristic zero. For each point $P$ in $\mathbb{P}^{3}$ let $V_{P}$ be the linear system on $C$ induced by the hyperplanes through $P$. By $V_{P}$ one maps $C$ onto a plane curve $C_{P}$, such a map can be seen as a projection of $C$ from $P$. If $P$ is not the vertex of a cone of bisecant lines, then $C_{P}$ will have only finitely many singular points; or to put it slightly different: The secant scheme $S_{P}=\left(V_{P}\right)_{2}^{1}$ parametrizing divisors in the second symmetric product $C_{2}$ that fail to impose independent conditions on $V_{P}$ will be finite. Hence each such point $P$ gives rise to a partition $\left\{a_{1} \geq a_{2} \geq \ldots \geq a_{k}\right\}$ of $\Delta(d, g)=\frac{1}{2}(d-1)(d-2)-g$, where the $a_{i}$ are the local multiplicities of the scheme $S_{P}$. If $P$ is the vertex of a cone of bisecant lines (for example if $P$ is a point of $C$ ), we set $a_{1}=\infty$. It is clear that the set of points $P$ with $a_{1} \geq 2$ is the surface $F$ of stationary bisecant lines (including some tangent lines); a generic point $P$ on $F$ gives a tacnodial $C_{P}$.

We give two results valid for all curves $C$. The first one describes the set of points $P$ with $a_{1} \geq 3$. The second result describes the set of points with $a_{1} \geq 4$.
\end{abstract}

1. Introduction. Let $C$ be a smooth non-degenerate integral curve of degree $d$ and genus $g$ in $\mathbb{P}^{3}$ over an algebraically closed field of characteristic zero. For each point $R$ in $\mathbb{P}^{3}$, let $V_{R}$ be the linear system on $C$ induced by the hyperplanes through $R$. If $R \notin C$, then $V_{R}$ maps $C$ into a plane, corresponding to a projection of $C$ from $R$. Let $C_{R}$ be the projected plane curve.

We now pose the following, rather unprecise problem: "Describe strata of $\mathbb{P}^{3} \backslash C$, such that the number and types of singularities of each $C_{R}$ are 'essentially unchanged' when $R$ varies in each stratum". It is well known that if $R$ is outside the following surfaces, then $C_{R}$ is a curve of degree $d$ with $\Delta(d, g)=\frac{1}{2}(d-1)(d-2)-g$ ordinary nodes of multiplicity 2 :

$F_{1}$ : The union of all tangent lines to $C$.

$F_{2}$ : The union of all $k$-secant lines to $C$, such that $k \geq 3$ (counted properly with multiplicity).

1991 Mathematics Subject Classification: Primary: 14H50; Secondary: 14N05.

The paper is in final form and no version of it will be published elsewhere. 
$F_{3}$ : The surface of stationary bisecant lines; that is: The closure of the union of all lines that connect two points of $C$, such that the tangent lines to $C$ at the two points are contained in the same plane. (This surface contains the tangent lines to $C$ at those points where the osculating plane intersects $C$ at least 4 times.)

We see that if $R$ is contained in $F_{1}$, then $C_{R}$ will have a cusp. If $R$ is contained in $F_{2}$, then $C_{R}$ will have a $k$-node, with $k \geq 3$ (in general). If $R$ is contained in $F_{3}$, then $C_{R}$ will have a tacnode or higher cusp.

Remark. Assume $C$ is contained in a cone that is a union of bisecant lines to $C$, with vertex $R$. Then $C_{R}$ will have degree less than $d$, and each of the generatrices of the cone will be a stationary bisecant line if $R$ is outside $C$. This is true because the tangent line to the cone stays constant along each generatrix.

Moreover we see that $C_{R}$ will have at least two cusps if $R$ is contained in a nodal edge of $F_{1}, 2$ trinodes (in general) if $R$ is contained in a nodal edge of $F_{2}$, and so on. Intuitively it therefore seems like the stratification mentioned above must in an intimate way be connected to stratifications of each of the 3 surfaces into: general stratum, nodal edge, possible cuspidal edge, intersection of such edges, triple locus, and so on. In addition we must study intersections of such strata for each pair of surfaces, and for all 3 surfaces simultaneously.

Instead of studying the complex interplay between all 3 surfaces, we will pose a simpler, but precise stratification problem. It will turn that this simplified problem will be intimately connected to a stratification of $F_{3}$.

For each $n$ denote by $C_{n}$ the $n$-th symmetric product of $C$. Let $R \in \mathbb{P}^{3}$, and let $\left(V_{R}\right)_{2}^{1}$ be the standard determinantal subscheme of $C_{2}$, which parametrizes those effective divisors of degree 2 that fail to impose linearly independent conditions on the linear system $V_{R}$. A point (divisor) $P+Q, P \neq Q$, of $\left(V_{R}\right)_{2}^{1}$ corresponds to a node of $C_{R}$, while a point $2 P$ corresponds to a cusp. For convenience we will denote $\left(V_{R}\right)_{2}^{1}$ by $S_{R}$.

We have the well-known formula if $S_{R}$ is finite:

$$
\sum_{D \in C_{2}} \delta_{R}(D)=\Delta(d, g)=\frac{1}{2}(d-1)(d-2)-g,
$$

where $\delta_{R}(D)$ is the multiplicity of the scheme $S_{R}$ at $D$. (See $[\mathrm{ACGH}]$, p. 351.) Hence each point $R$ with $S_{R}$ finite gives rise to a partition $\left\{a_{1} \geq a_{2} \geq \ldots \geq a_{k}\right\}$ of $\Delta(d, g)$, where the $a_{i}$ are the local multiplicities of the scheme $S_{R}$. If $S_{R}$ is infinite, for example, if $R \in C$, and in general if $R$ is the vertex of a cone of bisecants, then we set $a_{1}=\infty$. We denote by the stratum at infinity the set $\left\{R \mid a_{1}=\infty\right\}$. We now pose the following precise

Stratification Problem. For each fixed partition $\left\{a_{1} \geq a_{2} \geq \ldots \geq a_{k}\right\}$ of $\Delta(d, g)$ describe the set (stratum) of those $R$ that give rise to this partition.

EXAMPLE 1.1. The set corresponding to the partition $\{1 \geq \ldots \geq 1\}$ is obviously the complement of $F_{3}$ if this surface is non-empty, and the complement of $C$ if $F_{3}=\emptyset$. It is important to note that the surfaces $F_{1}$ and $F_{2}$ do not play any essential role in the last, precise stratification problem. A point $R$ on $F_{1}$ only gives rise to a point $2 P$ instead of $P+Q$ on $S_{R}$. A point $R$ on $F_{2}$ gives rise to a $k$-node of $C_{R}$, but this $k$-node is expected to give rise to $\left(\begin{array}{l}k \\ 2\end{array}\right)$ points, each of multiplicity 1 on $S_{R}$.

Furthermore it is clear that the closure of the stratum corresponding to the partition 
$\{2 \geq 2 \geq 1 \geq \ldots \geq 1\}$ is a nodal edge of $F_{3}$ if non-empty. Our goal is to describe the union of the strata with $a_{1} \geq 3$, and the union of the strata with $a_{1} \geq 4$, in form of results valid for all smooth, non-degenerate integral curves in $\mathbb{P}^{3}$ (Theorems 2.1 and 2.2). We prove these results in Sections 3 and 4. In Section 4 we also introduce a somewhat new blend of techniques, suitable for proving local results about parameter spaces (Result 4.1). In Section 5 we give some remarks to illustrate the significance of the results of Section 2 . It is our hope that not only our main results, but also the proofs of them, will give some insight into the geometry of space curves.

R e m ark 1.2. Let $V$ be a base point free linear system of rank 3 on a smooth curve $C$, defining a birational map $\phi$ onto a plane curve $\bar{C}$. Let $Q$ be a (singular) point of $\bar{C}$. Then the $\delta$-invariant, see for example [Te], of $\bar{C}$ at $Q$ is equal to the sum of multiplicities of the scheme $V_{2}^{1}$ at the points $P_{1}+P_{2}$ and $2 P$, such that $\phi\left(P_{1}\right)=\phi\left(P_{2}\right)=Q$, and $\phi(P)=Q$. This is stated without proof in [LP-I], p. 199. We remark that an easy proof follows from comparing the calculations of the $\delta$-invariant of $\bar{C}$ at $Q$ on pp. $611-612$ of [Te], and the calculations of the sum above, performed along the lines of $\S 6$ of [LP-I].

Hence our stratification of $\mathbb{P}^{3} \backslash C$ in terms of partitions of $\Delta(d, g)$ is really a stratification of $\mathbb{P}^{3} \backslash C$ due to the $\delta$-invariants of the branches and pairs of branches of the singularities of the plane curves $C_{R}$ (when $C$ is birational to $C_{R}$ ).

Remark 1.3. Let $X \subseteq C_{2} \times \mathbb{P}^{3}$ be the incidence $\{(D, R) \mid R$ is contained in "the line spanned by $D$ " $\}$. ("The line spanned by $D$ " is $\overline{P_{1} P_{2}}$ if $D=P_{1}+P_{2}$, for $P_{1} \neq P_{2}$ and the tangent line to $C$ at $P$ if $D=2 P$.) $X$ is clearly a smooth 3 -fold, being a $\mathbb{P}^{1}$-bundle over $C_{2}$. Let $f$ be the second projection map from $X$ on $\mathbb{P}^{3}$. We now define strata $S_{i}(f)$ :

$$
S_{i}(f)=\left\{x \in X \mid \text { the map }(d f)_{x}: T(X)_{x} \rightarrow T\left(\mathbb{P}_{3}\right)_{f(x)} \text { has rank } 3-i\right\} .
$$

Equivalently, $x \in S_{i}(f)$ iff the tangent space of the scheme theoretical fibre of $f(x)$ at $x$ is equal to $i$. See for example p. 231, and Proposition 2.4, p. 233, of [Ro]. See also [Th] and $[\mathrm{Bo}]$ for similar strata for differentiable maps of real manifolds.

We also see that the condition " $R$ is contained in the line spanned by $D$ ", describing the incidence $X$, could be reformulated as: $D \in\left(V_{R}\right)_{2}^{1}$. We therefore have a natural identification: $f^{-1}(R)=\left(V_{R}\right)_{2}^{1} \subseteq C_{2}$. Since $\operatorname{dim} C_{2}=2$, we have $S\left(f_{3}\right)=\emptyset$. From Theorem 2.5 of [LP-II] it follows that the tangent space dimension of $\left(V_{R}\right)_{2}^{1}$ at $D$ is 2 iff "the line spanned by $D$ " contains $R$ and is tangent to $C$ at $P_{1}$ and $P_{2}$, in the case $D=P_{1}+P_{2}$. In the case $D=2 P$ the corresponding condition is that all planes containing $P$ and $R$ intersect $C$ at least 4 times at $P$ (so $\overline{P R}$ is a higher flex tangent at $P$ ). One can show that $a_{1} \geq 4$ for the $R$ appearing in these cases (that is: for $R \in f\left(S_{2}(f)\right)$ ). Moreover there is a natural identification between the higher order singularity subschemes $S_{1}^{(q)}(f)$ of $X$, defined in [Ro], and

$\left\{(D, R) \mid\left(V_{R}\right)_{2}^{1}\right.$ has tangent space dimension 1 and multiplicity at least $q+1$ at $\left.D\right\}$, for $q=1,2, \ldots$. Hence we see that (if $R \notin$ stratum at infinity, then): $a_{1}=2$ for $R \in$ $f\left(S_{1}(f) \backslash S_{1}^{(2)}(f)\right), a_{1}=3$ for $R \in f\left(S_{1}^{(2)}(f) \backslash S^{(3)}(f)\right)$, and $a_{1} \geq 4$ for $R \in f\left(S_{2}(f) \cup\right.$ $\left.S_{1}^{(3)}(f)\right)$. We then see that the stratification problem in terms of the $a_{i}$ is closely connected to the theory of higher order singularity subschemes, for example as described in [Ro].

For a generic map $g$ between 2 smooth 3 -folds, for example, in the sense of [Bo], we have $S_{2}(g)=\emptyset$ and $\operatorname{dim} S_{1}^{(q)}(g)=3-q$, for $q=1,2, \ldots$. Moreover one can (for such 
generic $g$ ) identify $S_{1}^{(q)}(g)$ with $S_{1}\left(f \mid S_{1}^{(q-1)}(g)\right)$, for $q \geq 2$, where $S_{1}^{(1)}(f)$ means $S_{1}(f)$. In other words; the $q$-th higher singularity locus of $g$ is the singularity locus of $g$, restricted to the $(q-1)$-th singularity locus, for $q \geq 2$.

If we pretend that our map $f: X \rightarrow \mathbb{P}^{3}$ is generic in the sense above, then we see that the last statement is very close to saying that

$$
\begin{aligned}
& \left\{R \mid a_{1}=3\right\} \text { is the cuspidal edge of the surface } F_{3} \text {, and } \\
& \left\{R \mid a_{1}=4\right\} \text { is }\left\{\text { cusps of the curve }\left\{R \mid a_{1}=3\right\}\right\} .
\end{aligned}
$$

In reality, however, our map $f: X \rightarrow \mathbb{P}^{3}$ is not generic. The fibres of $f$ over points of $C$ are not even finite. Moreover, we want results valid for all smooth curves $C \subseteq \mathbb{P}^{3}$, and thus we do not want to rule out the possibility of "unexpected" phenomena like bitangents, higher flexes, points outside $C$ being vertices of cones of bitangents, and so on. Therefore, we are not using the theory of generic maps and higher singularity schemes of these, as in $[\mathrm{Th}]$, [Bo], [Ro]. Instead we are using some local techniques taken from [LP-I], [LP-II], [MS]. It is therefore natural that we obtain, in Theorems 2.1 and 2.2, a picture which is more complicated than for that of a generic map. Our results do however imply that for what we in rough terms would call "a generic curve $C$ of degree $d$ and genus $g$ ( $d$ and $g$ high enough)", the sets $\left\{R \mid a_{1}=3\right\}$ and $\left\{R \mid a_{1}=4\right\}$ "essentially behave as sets $S_{1}^{(2)}(g)$ and $S_{1}^{(3)}(g)$, respectively, of a generic map $g$ between two 3 -folds". For more details, see Remarks 5.1 and 5.2 .

For such a generic map the union of $S_{1}^{(m)}(g)$, for $m \geq 4$ (as well as $S_{2}(g)$ ), in our situation corresponding to $\left\{R \mid a_{1} \geq 5\right\}$, is empty. Since we do not assume that $f$ is in any way generic, the sets $\left\{R \mid a_{1}=m\right\}, m \geq 5$, may be non-empty, and one could try to give results describing the sets $\left\{R \mid a_{1} \geq m\right\}$, for all $m$, valid for all smooth space curves. Because of the complexity of the calculations involved, we have chosen to stop at $m=4$. In particular we have problems with describing in a compact way which points $R$ on bitangent lines and higher flexes (points of $\left.f\left(S_{2}(f)\right)\right)$ that have $a_{1} \geq m$, for fixed $m \geq 5$.

This paper can be read as a continuation of $\S 5$ in $[\mathrm{LP}-\mathrm{II}]$, and is the "more detailed study" referred to at the end of [LP-II].

I am grateful to Ignacio Sols for asking me the question raised in Remark 5.4: Which curves in $\mathbb{P}^{3}$ are such that the monodromy group of the points of $S_{R}$, as $R$ varies over $\mathbb{P}^{3}$, is the full symmetric group?

I am also very grateful to Alf Aure for making me aware of the results from [BM] quoted in Remark 5.4. These results give a partial answer to that question.

The work of this paper has been supported in part by the Norwegian Research Council for Science and the Humanities. We thank Brandeis University where the author was a visitor while receiving this support.

I thank the organizers of the workshop Parameter Spaces at the Banach Center in Warsaw, 1994, for a pleasant and rewarding conference, and for retyping the manuscript.

2. Main results. Let $C$ be as in the introduction. Let $B(C)$ be the closure in the dual space $\check{\mathbb{P}}^{3}$ of the set of points that parametrize planes that contain the tangent lines of $C$ at 2 distinct points of $C$.

$B(C)$ may be empty, as when $C$ is a twisted cubic curve. $B(C)$ may be reducible, as when $C$ is the complete intersection of 2 quadrics $(d=4, g=1)$. In this case, for a 
general choice of quadrics, $B(C)$ will consist of 4 degenerate (conic) components, each of them parametrizing tangent planes to some quadric cone that contains $C$.

For a reduced, non-degenerate curve $S$ in $H$, where $H$ is $\mathbb{P}^{3}$ or $\check{\mathbb{P}}^{3}$, denote by $D(S)$ the strict dual curve of $S$. The curve $D(S)$ parametrizes the closure, in the dual space $\check{H}$, of the set of those points that parametrize the osculating planes to $S$ at its smooth points. $D(S)$ will be a non-degenerate curve in $\check{H}$, and it will be irreducible if $S$ is so.

Give each of the components of $B(C)$ a reduced structure. We are now ready to give our 2 main results (Theorems 2.1 and 2.2).

THEOREM 2.1.

$$
\left\{R \in \mathbb{P}^{3} \mid a_{1} \geq 3\right\}=\mathcal{C} \cup C,
$$

where $\mathcal{C}$ is the union of the subsets a)- $\mathrm{f})$ of $\mathbb{P}^{3}$ :

a) The union of the $D\left(S_{i}\right)$ for each irreducible non-degenerate component $S_{i}$ of $B(C)$.

b) The set of points that parametrize planes in $\check{\mathbb{P}}^{3}$ that contain a degenerate component of $B(C)$.

c) All flex tangent lines $C$ at some point $P$.

d) All lines connecting 2 distinct points $P$ and $Q$ of $C$, such that there is a plane $H$ that intersects $C$ at least 3 times at $P$ and $Q$.

e) All lines connecting 2 distinct points $P$ and $Q$ of $C$, such that there is a plane $H$ that intersects $C$ at least 3 times at $P$ and at least 2 times at $Q$, and such that $L=\overline{P Q}$ is tangent to $C$ at $P$.

f) All tangent lines to $C$ at points $P$, such that there is a plane $H$ that intersects $C$ at least 6 times at $P$.

Remark. The set of points of b) may be empty, or finite as in the case with the elliptic quartic curve that is the complete intersection of 2 quadrics, or infinite as in the case of a curve possessing a bitangent line. This line is then contained in the infinite set. We see that each component of $\mathcal{C}$ is at most 1-dimensional, since a curve $C$ has only finitely many bitangents, flexes, tangential trisecants, hyperosculating planes, and biosculating planes.

Remark. Each $D\left(S_{i}\right)$ of part a) is a cuspidal edge of the surface $F_{3}$ of Section 1.

THEOREM 2.2.

$$
\left\{R \in \mathbb{P}^{3} \mid a_{1} \geq 4\right\}=M \cup C,
$$

where $M$ is the union of the subsets a1) - $\mathrm{f} 2)$ of $\mathbb{P}^{3}$.

a1) The set of cusps of the non-linear components of $C$.

b1) The set of points that parametrize planes in $\mathbb{P}^{3}$ that contain a degenerate component of $B(C)$.

c1) The union of all flex lines such that some plane intersects $C$ at least 5 times at the flex point $P$.

d1) All lines that contain 2 distinct points $P$ and $Q$, such that there is a plane $H$ that intersects $C$ at least 4 times at both $P$ and $Q$.

d2) For each line $L$ connecting 2 points $P$ and $Q$ such that there is a plane $H$ that intersects $C$ exactly 3 times at $P$ and $Q$, and such that $L$ is not a tangent line to $C$ at any of the points $P$ and $Q$ :

$A$ set of exactly 2 points. Every such point $R$ is:

$A$ vertex of a cone of bisecant lines

or $A$ point where $L$ meets and is tangent to a non-linear component of $\mathcal{C}$. 
In any case $R$ is the vertex of a cubic cone $N$, such that $L \nsubseteq \operatorname{Sing} N$, and such that $N$ intersects $C$ at least 4 times at $P$ and $Q$.

e1) The union of all tangent lines to $C$ at some point $P$, reintersecting $C$ at some other point $Q$, such that there is a plane $H$ that intersects $C$ at least 4 times at $P$ and at least 2 times at $Q$.

f1) The union of all tangent lines $L$ at points $P$, such that some plane intersects $C$ at least 8 times at $P$.

f2) For each tangent line that is not a flex tangent line, and where the osculating plane intersects $C$ exactly 6 times at the point $P$ of tangency:

$A$ set of exactly one point. This point $R$ is:

$A$ vertex of a cone of bisecants

or $A$ point where $L$ meets and is tangent to a non-linear component of $\mathcal{C}$.

In any case $R$ is the vertex of a cubic cone $N$, such that $L \nsubseteq \operatorname{Sing} N$, and such that $N$ intersects $C$ at least 8 times at $P$.

3. Proof of main results. We will sketch an argument for proving Theorems 2.1 and 2.2 simultaneously. The proof will be completed in Section 4 . By definition $a_{1}=\infty$ if $R \in C$, so we can safely disregard the case where $R \in C$. Hence we assume $R \notin C$. Also it is clear from Example 1.1 that $a_{1}=1$ if $R$ is not contained in the surface $F_{3}$ of Section 1 . Moreover it is clear that the sets $\mathcal{C}$ and $M$ of Theorems 2.1 and 2.2 are contained in $F_{3}$. Hence we may assume that $R$ is contained in $F_{3}, R \notin C$, and $a_{1} \geq 2$.

Convention. If we write $D=P+Q$, we implicitly assume that $P \neq Q$.

Definition. We denote by $I\left(p, M_{1} \cap M_{2}\right)$ the intersection number at a point $p$ of 2 varieties $M_{1}$ and $M_{2}$ of complementary dimension in $\mathbb{P}^{r}$, for $r \geq 2$.

Since $a_{1} \geq 2$, there is at least one point (divisor) $D$ in the symmetric product $C_{2}$ such that the multiplicity of $S_{R}$ at $D$ is at least 2 .

If $D=P+Q$, then we denote by $L(D)$ or $L$ the line $\overline{P Q}$. This line will be a stationary bisecant line to $C$. If $L$ is not tangent to $C$ at both $P$ and $Q$, then we denote by $H(D)$ or $H$ the unique plane $H$ containing $L$, such that $I(P, C \cap H) \geq 2$, and $I(Q, C \cap H) \geq 2$.

If $D=2 P$, we denote by $L$ or $L(D)$ the tangent line to $C$ at $P$. If $I\left(P, C \cap H^{\prime}\right) \leq 3$ for some plane $H^{\prime}$ containing $L$, we denote by $H$ or $H(D)$ the unique plane such that $I(P, C \cap H) \geq 4$. We note that both in the case $D=P+Q$, and in the case $D=2 P$, the point $R$ will be contained in $L$. Furthermore $D$ is a point of multiplicity at least 2 of the scheme $S_{R}$, for all $R \in L(D)$. By convention we say that $D$ is a point of multiplicity $\infty$ of $S_{R}$ if $S_{R}$ is non-isolated at $D$.

From [LP-II], $\S 5$, it follows that there is a one-dimensional family of stationary bisecants, if any at all. Moreover each component of this family envelopes a curve in $\mathbb{P}^{3}$ or gives rise to a cone of bisecant lines. The curves that arise this way are exactly the $D\left(S_{i}\right)$ described in Theorem 2.1.a). Moreover there is a subcurve $\mathcal{S}^{\prime}$ of the second symmetric product $C_{2}$ such that $\mathcal{S}^{\prime}$ parametrizes divisors $D$ such that there is a point $R$ outside $C$ with the multiplicity of $S_{R}$ at $D$ at least 2 . The family of stationary bisecants, viewed as a curve $\mathcal{S}$ in the Grassmannian $G(1,3)$, arises as the image of $\mathcal{S}^{\prime}$ under the map $D \rightarrow L(D)$. For each non-degenerate component $S_{i}$ of $B(C)$ we have a so called complete triple $\left(D\left(S_{i}\right), \mathcal{S}_{i}, S_{i}\right) \subseteq \mathbb{P}^{3} \times G(1,3) \times \check{\mathbb{P}}^{3}$, where $\mathcal{S}_{i}$ is a component of $\mathcal{S}$. For each branch $\mathcal{S}^{\prime}$ through $D$, take its associated (image) branch of $\mathcal{S}$ through the point $\ell \in G(1,3)$ representing $L(D)$. For each such branch, take the point on $L(D) \in \mathbb{P}^{3}$ enveloped by 
the branch. (The tangent line to $\mathcal{S}$ at $\ell$ in $\mathbb{P}^{5}$ will be globally contained in $G(1,3)$ as an intersection between the dual $\beta$-plane of $H(D)$ and the $\alpha$-plane representing all lines through some fixed point of $L$. This is the point referred to as "enveloped by the branch of $\mathcal{S}$ ".) Each such point will be a point of some $D\left(S_{i}\right)$ or a vertex of a cone of bisecant lines to $C$. For each fixed $D$ take the union of the points enveloped by the branches in question.

We denote by $M(D)$ this union of points in $\mathbb{P}^{3}$. In order to prove Theorems 2.1 and 2.2 it will be enough to prove that one of the following 7 possibilities occurs for each divisor $D \in C_{2}$ appearing with multiplicity at least 2 for some scheme $S_{R}$, with $R \notin C$ :

(i) $\quad D=P+Q, \quad I(P, C \cap H(D))=I(Q, C \cap H(D))=2$.

$L(D)$ is tangent to $C$ neither at $P$ nor at $Q$, and for $R \in L(D) \backslash C$ we have

$R \in M(D) \Leftrightarrow S_{R}$ has multiplicity at least 3 at $D$.

We also have: Due to some branch of $\mathcal{S}^{\prime}$ through $D, R$ is either a cusp of some nondegenerate curve $D\left(S_{i}\right)$ or a vertex of a cone of bisecants $\Leftrightarrow S_{R}$ has multiplicity at least 4 at $D$.

(ii) $\quad D=2 P, \quad P$ is not a flex, and $I(Q, C \cap H(D))=4$.

For $R \in L(D) \backslash C$ we have the same equivalences as in (i).

(iii) $\quad S_{R}$ has multiplicity exactly 2 at $D$ for all $R \in L(D) \backslash C$

and: All points of $M(D)$ are contained in $C$,

and: In case $D=P+Q, L(D)$ is neither a bitangent line to $C$ nor a line satisfying d) or e) of Theorem 2.1 .

In case $D=2 P, L(D)$ satisfies neither c) nor f) of Theorem 2.1.

(iv) $\quad S_{R}$ has multiplicity exactly 3 at $D$ for all $R \in L(D) \backslash C$.

and: All points of $M(D)$ are contained in $C$.

and: In case $D=P+Q, L(D)$ satisfies d) or e) of Theorem 2.1, is not bitangent line, and satisfies neither $\mathrm{d} 1$ ) nor e1) of Theorem 2.2 .

In case $D=2 P, L(D)$ satisfies c) or f) of Theorem 2.1, but neither c1) nor f1) of Theorem 2.2 .

(v) $\quad S_{R}$ has multiplicity at least 4 at $D$ for all $R \in L(D)$

and: In case $D=P+Q, L(D)$ is bitangent to $C$ at $P$ and $Q$, or $L(D)$ satisfies $\mathrm{d} 1)$ or e1) of Theorem 2.2 .

In case $D=2 P, L(D)$ satisfies $\mathrm{c} 1$ ) or $\mathrm{f} 1$ ) of Theorem 2.2 .

(vi) We are in the situation described in Theorem 2.1.d) and Theorem 2.2.d2), that is: $L(D)$ is tangent to $C$ neither at $P$ nor at $Q$, and $I(P, C \cap H(D))=$ $I(Q, C \cap H(D))=3$, and $S_{R}$ has multiplicity at least 3 at $D$ for all $R \in L(D)$, and at least 4 for $R$ described in Theorem 2.2.d2).

(vii) We are in the situation described in Theorem 2.1.f) and Theorem 2.2.f2), that is: $P$ is not a flex, $I(P, C \cap H(D))=6$ and $S_{R}$ has multiplicity at least 3 at $D$ for all $R \in L(D)$, and at least 4 for $R$ described in Theorem 2.2.f2.).

For a given $D$ of multiplicity at least 2 in some scheme $S_{R}$ we now list 17 possibilities for $D$. It is immediately clear that one of these 17 possibilities must occur for given $D$ as mentioned. The proof of the main results will consist of sketching how in each of these 17 cases: 1$), \ldots, 17)$. we are in one of the cases (i),..,(vii) above.

1) $D=P+Q \cdot L(D)$ is tangent neither at $P$ nor at $Q$.

$I(P, C \cap H(D))=I(Q, C \cap H(D))=2$.

2) $D=P+Q$. $L(D)$ is tangent at $P$, but not at $Q$. 
$I(P, C \cap H(D))=I(Q, C \cap H(D))=2$.

3) $D=P+Q$. $L(D)$ is tangent at both $P$ and $Q$.

4) $D=P+Q$. $L(D)$ is tangent neither at $P$ nor at $Q$.

$I(P, C \cap H(D)) \geq 3 . \quad I(Q, C \cap H(D))=2$.

5) $D=P+Q . L(D)$ is tangent at $P$, but not at $Q$. $I(P, C \cap H(D))=3$.

6) $D=P+Q \cdot L(D)$ is tangent at $Q$, but not at $P$. $I(P, C \cap H(D)) \geq 3 . \quad I(Q, C \cap H(D))=2$.

7) $D=P+Q \cdot L(D)$ is tangent at $P$, but not at $Q$. $I(P, C \cap H(D)) \geq 4$.

8) $D=P+Q$. $L(D)$ is tangent neither at $P$ nor at $Q$. $I(P, C \cap H(D))=I(Q, C \cap H(D))=3$.

9) $D=P+Q$. $L(D)$ is tangent neither at $P$ nor at $Q$. $I(P, C \cap H(D)) \geq 4 . \quad I(Q, C \cap H(D))=3$.

10) $D=P+Q . I(P, C \cap H(D)) \geq 4 . \quad I(Q, C \cap H(D)) \geq 4$.

11) $D=2 P$. $P$ not a flex. $I(P, C \cap H(D))=4$.

12) $D=2 P$. $P$ not a flex. $I(P, C \cap H(D))=5$.

13) $D=2 P$. $P$ not a flex. $I(P, C \cap H(D))=6$.

14) $D=2 P$. $P$ not a flex. $I(P, C \cap H(D))=7$.

15) $I(P, C \cap H) \geq 8$ for some plane $H$.

16) $D=2 P$. $P$ is a flex of $C . I(P, C \cap H(D))=4$.

17) $D=2 P$. $P$ is a flex of $C . I(P, C \cap H(D)) \geq 5$ for some plane $H$.

What we will sketch, is a proof of the statements indicated in the following table:

DIVISORS $P+Q$

\begin{tabular}{|c|c|c|}
\hline Case & Case & $M(D)$ \\
\hline 1 & (i) & $\left\{R_{1}\right\}, R_{1} \notin\{P, Q\}$ \\
\hline 2 & (iii) & $\{P\}$ \\
\hline 3 & $(\mathrm{v})$ & Depends on more information \\
\hline 4 & (iii) & $\{Q\}$ \\
\hline 5 & (iv) & $\{P\}$ \\
\hline 6 & (iii) & $\{Q\}$ \\
\hline 7 & $(\mathrm{v})$ & $\{P\}$ \\
\hline 8 & (vi) & $\left\{R_{1}, R_{2}\right\}, R_{1} \neq R_{2}, R_{i} \notin\{P, Q\}, i=1,2$ \\
\hline 9 & (iv) & $\{Q\}$ \\
\hline 10 & $(\mathrm{v})$ & Depends on more information \\
\hline
\end{tabular}

DIVISORS $2 P$

\begin{tabular}{|c|l|l|}
\hline Case & Case & $M(D)$ \\
\hline 11 & (ii) & $\left\{R_{1}\right\}, R_{1} \neq P$ \\
12 & (iii) & $\{P\}$ \\
13 & (vii) & $\left\{P, R_{1}\right\}, R_{1} \neq P$ \\
14 & (iv) & $\{P\}$ \\
15 & (v) & Depends on more information \\
16 & (iv) & $\{P\}$ \\
17 & (v) & Depends on more information \\
\hline
\end{tabular}


In order to proceed, we shall frequently use the following results, among which Lemma 3.1 is standard, and Lemma 3.2 follows for example from [LP-I], $\S 6$.

LEMma 3.1. Let $V$ be a linear system of (affine) rank 3 on $C$, spanned by sections $S_{1}, S_{2}, S_{3}$. Let

$$
S_{i}=\sum_{k=0}^{\infty} a_{i, j, k} t_{j}^{k}
$$

be local parametrizations of $S_{i}$ at $P_{j}$, for $i=1,2,3$, and $j=1,2$. Then the completion of the local ring of the secant scheme $V$ at $D=P_{1}+P_{2}$ is $k\left[\left[t_{1}, t_{2}\right]\right] / \mathcal{I}$, where $\mathcal{I}$ is generated by the 2-minors of

$$
\left[\begin{array}{ccc}
\sum a_{1,1, k} t_{1}^{k} & \sum a_{2,1, k} t_{1}^{k} & \sum a_{3,1, k} t_{1}^{k} \\
\sum a_{1,2, k} t_{2}^{k} & \sum a_{2,2, k} t_{2}^{k} & \sum a_{3,2, k} t_{2}^{k}
\end{array}\right]
$$

The $t_{i}$ are regarded as indeterminates.

LEMma 3.2. Let $V$ be a linear system of (affine) rank 3 on $C$, spanned by sections $S_{1}, S_{2}, S_{3}$. Let

$$
S_{i}=\sum_{k=0}^{\infty} a_{i, k} t^{k}, \text { for } i=1,2,3
$$

be local parametrizations of $S_{i}$ at $P$, for $i=1,2,3$. Then the completion of the local ring of the secant scheme $V_{2}$ at $2 P$ is $k\left[\left[s_{1}, s_{2}\right]\right] / J$, where $J$ is generated by the 2 -minors of

and

$$
\left[\begin{array}{ccc}
\sum a_{1, k} W_{k} & \sum a_{2, k} W_{k} & \sum a_{3, k} W_{k} \\
\sum a_{1, k} W_{k-1} & \sum a_{2, k} W_{k-1} & \sum a_{3, k} W_{k-1}
\end{array}\right]
$$

$$
W_{j}=\sum_{i=0}^{[j / 2]}\left(\begin{array}{c}
j-i \\
i
\end{array}\right) s_{1}^{j-2 i} \cdot s_{2}^{i} .
$$

Here the $s_{i}$ are regarded as indeterminates, and $[x]$ means the integral part of the real number $x$.

Cases 1) And 2). Let $\mathbb{P}^{3}=\operatorname{Proj} k[X, Y, Z, W]$, and let $P=(0,0, p, 1), Q=(0,0, q, 1)$, and let $t, u$ be local parameters of $C$ at $P, Q$ respectively. Let

$$
\begin{array}{lll}
X=\alpha_{1} t+\alpha_{2} t^{2}+\ldots & & X=\mu_{1} u+\mu_{2} u^{2} \\
Y=\quad \beta_{2} t^{2}+\ldots & \text { and } \quad & Y=\nu_{2} u^{2}+\ldots \\
Z=p+t & & Z=q+u \\
W=1 & W=1
\end{array}
$$

be local parametrizations of $C$ at $P$ and $Q$, respectively. One sees that the equations of $L(D)$ and $H(D)$ are $X=Y=0$ and $Y=0$, respectively.

$$
\beta_{2} \nu_{2} \neq 0, \text { since } I(P, C \cap H(D))=I(Q, C \cap H(D))=2,
$$

and we are in Case 1 ) or 2 ), depending on whether $a_{1} \mu_{1} \neq 0$, or not. Either $a_{1} \neq 0$, or $\mu_{1} \neq 0$, since $L(D)$ is not a bitangent line.

We will study the curve $B(C)$ at the point $h$ in $\check{P}^{3}$ corresponding to $H(D)$. Let Spec $k\left[b_{1}, b_{2}, b_{3}\right]$ be an affine piece of $\check{P}^{3}$, where the point $\left(b_{1}, b_{2}, b_{3}\right)$ corresponds to the plane $Y+b_{1} X+b_{2} Z+b_{3} W=0$. From Theorem 2.2 and Formula (3.3) of [MS] it follows that the completion of the local ring of the branch of $B(C)$ in question at $h=(0,0,0)$ is:

$$
\hat{\mathcal{O}}=k\left[\left[b_{1}, b_{2}, b_{3}\right]\right] /\left(f_{1}(\underline{b}), f_{2}(\underline{b})\right)
$$


for 2 explicit power series $f_{1}$ and $f_{2}$ in the indeterminates $b_{1}, b_{2}, b_{3}$. Let $\underline{m}=\left(b_{1}, b_{2}, b_{3}\right)$. We then have

$$
\begin{aligned}
& f_{1} \equiv\left(b_{3}+p b_{2}\right)-\frac{1}{4 \beta_{2}}\left(b_{2}+\alpha_{1} b_{1}\right)^{2}+\frac{\alpha_{2}}{4 \beta_{2}^{2}}\left(b_{2}+\alpha_{1} b_{1}\right)^{2} b_{1}-\frac{\beta_{3}}{8 \beta_{2}^{3}}\left(b_{2}+\alpha_{1} b_{1}\right)^{3} \\
& f_{2} \equiv\left(b_{3}+q b_{2}\right)-\frac{1}{4 \nu_{2}}\left(b_{2}+\mu_{1} b_{1}\right)^{2}+\frac{\mu_{2}}{4 \nu_{2}^{2}}\left(b_{2}+\mu_{1} b_{1}\right)^{2} b_{1}-\frac{\nu_{3}}{8 \nu_{2}^{3}}\left(b_{2}+\nu_{1} b_{1}\right)^{3} .
\end{aligned}
$$

Both congruences are modulo $\underline{m}^{4}$. Since $p \neq q$, the linear parts are independent, and we see that the tangent space of $B(C)$ is cut out by: $b_{3}=b_{2}=0$, and that the canonical image of $b_{1}$ can be taken as a local parameter of $B(C)$ at $h$. We then have that the osculating plane of $B(C)$ at $h$ has the equation: $i_{0} b_{2}+j_{0} b_{3}=0$, for some $\left(i_{0}, j_{0}\right) \in \mathbb{P}^{1}$.

(If we allow $L(D)$ to be a bitangent, that is: $\alpha_{1}=\mu_{1}=0$, we now see that both $b_{2}$ and $b_{3}$ are of order at least 3 in $b_{1}$ in $\hat{\mathcal{O}}$, and $B(C)$ has a flex.) Since we have assumed that $L(D)$ is not bitangent at $P$ and $Q$, then $\nu \neq 0$, or $\mu \neq 0$, and we see that

$$
\left(q \nu_{2} \alpha_{1}^{2}-p \beta_{2} \mu_{1}^{2}\right) b_{2}+\left(\nu_{2} \alpha_{1}^{2}-\beta_{2} \mu_{1}^{2}\right) b_{3}=0
$$

is the equation of the osculating plane of $B(C)$ at $h$ (and that $h$ is not a flex of the (branch of) $B(C)$ (that we study). Dually this osculating plane corresponds to the point

$$
(X, Y, Z, W)=\left(0,0, q \nu_{2} \alpha_{1}^{2}-p \beta_{2} \mu_{1}^{2}, \nu_{2} \alpha_{1}^{2}-\beta_{2} \mu_{1}^{2}\right) \in \mathbb{P}^{3} .
$$

Call this point $R_{1}$. We have now established

$$
M(D)=\left\{R_{1}\right\} .
$$

We see that in Case 2$)$ we have $\left(\alpha_{1}=0\right)$

$$
R_{1}=\left(0,0,-p_{2} \beta_{2} \mu_{1}^{2},-\beta_{2} \mu_{1}^{2}\right)=P
$$

and that if $\alpha_{1} \mu_{1} \neq 0$, then $R_{1} \neq P, R_{1} \neq Q$.

We will interpret $R_{1}$ as the unique point $R$ on $L(D)$, such that $S_{R}$ has multiplicity at least 3 at $D$. Let $R=(0,0, i, j)$, for $(i, j) \in \mathbb{P}^{1}$. Then the desired interpretation of $R_{1}$ follows from direct computations using Lemma 3.1 for the case:

$$
S_{1}=j Z-i W, \quad S_{2}=X, \quad S_{3}=Y .
$$

This shows that in Case 2) we are in Case (iii).

Re mark 3.3

a) It is also interesting to note that $R_{1}$ is the only point $R$ on $L$ such that there is a quadric cone $N$ with vertex $R$, not containing $L$ in its singular locus, such that $N$ intersects $C$ at least 3 times at $P$ and $Q$.

b) The results of the computations done so far in Case 1) follow directly from the discussion in [LP-II], §5. The reason why we have started from scratch instead of applying $[\mathrm{LP}-\mathrm{II}]$ is the rest of the proof in Case 1 ).

We finish the proof in Case 1): Let $R_{1}=\left(0,0, i_{0}, j_{0}\right)$ where $i_{0}=q \nu_{2} \alpha_{1}^{2}-p \beta_{2} \mu_{1}^{2}$, $j_{0}=\nu_{2} \alpha_{1}^{2}-\beta_{2} \mu_{1}^{2}$, and $\alpha_{1} \mu_{1} \nu_{2} \beta_{2} \neq 0$. The osculating plane of $B(C)$ at $h$ has the equation

$$
i_{0} b_{2}+j_{0} b_{3}=0 \text {. }
$$

By duality $R_{1}$ is a cusp of (the branch of) $D(B(C)$ ) or a vertex of a cone of bisecants if and only if the osculating plane at $h$ intersects $B(C)$ at least 4 times. From the explicit 
description of the power series $f_{1}$ and $f_{2}$ above it follows that $i_{0} b_{2}+j_{0} b_{3}$ is congruent to

$$
\begin{aligned}
L\left(b_{1}\right)=\frac{\alpha_{1} \mu_{1}}{8(q-p) \beta_{2}^{3} \nu_{2}^{3}} & {\left[\alpha_{1}^{2} \mu_{1} \beta_{2} \nu_{2}^{2}+\alpha_{1} \mu_{1}^{2} \beta_{2}^{2} \nu_{2}-\alpha_{1}^{3} \beta_{2} \nu_{2}^{2}\right.} \\
& -\mu_{1}^{3} \beta_{2}^{2} \nu_{2}+\alpha_{1}^{2} \mu_{1} \beta_{3} \nu_{2}^{2}(p-q)-\alpha_{1} \mu_{1}^{2} \beta_{2}^{2} \nu_{3}(p-q) \\
& \left.+2 \alpha_{1} \beta_{2}^{2} \mu_{1} \mu_{2} \nu_{2}(p-q)-2 \alpha_{1} \alpha_{2} \mu_{1} \beta_{2}(p-q)\right] \cdot b_{1}^{3}
\end{aligned}
$$

modulo $\underline{m}^{4}$ in the ring $\hat{\mathcal{O}}$. Hence $R_{1}$ is a cusp in Case 1 ) if and only if the contents of the parenthesis is zero. On the other hand it follows from direct calculations using Lemma 3.1 that the contents of the parenthesis is zero if and only if the multiplicity of the scheme $S_{R_{1}}$ at $D$ is at least 4 .

R e mark. Let $N(X, Y, Z, W)$ be the defining polynomial of the cone $N$ of Remark 3.3.a). Then $S_{R_{1}}$ has multiplicity at least 4 at $D$ if and only if there exists a scalar $b$ such that the cubic with equation

$$
N(X, Y, Z, W)\left(j_{0} Z-i_{0} W\right)+b X^{3}=0
$$

intersects $C$ at least 4 times at $P$ and $Q$. This description is more handy than doing calculations from scratch in this case.

The proof is now complete in Cases 1) and 2). We remark that $L\left(b_{1}\right)=0$ in Case 2) since $\alpha_{1} \mu_{1}=0$ then. Hence $P$ will be a cusp of, and $L(D)$ a cusp tangent to, $D(B(C))$ in Case 2).

CAses 3), 7) AND 10). Along with Cases 15) and 17) below these are the easiest cases. We see directly that in Cases 3), 7), 10) we are in the cases b1), e1) and d1), respectively, of Theorem 2.2. All we have to do, in order to show that we are in Case (v), is to use Lemma 3.1 to prove that $S_{R}$ has multiplicity at least 4 at $D$, for all $R \in L(D)$. We skip the calculations here. Case 3$)$ is treated in $[\mathrm{Pr}]$.

CASES 15) AND 17). We see directly that we are in the situations described in parts f1) and c1) of Theorem 2.2, respectively. To prove that we are in Case (v), we must use Lemma 3.2 to show that the multiplicity of $S_{R}$ at $D$ is at least 4 for all $R \in L(D)$. We treat Case 17). One treats Case 15) in a similar way. We choose parametrizations:

$$
W=1, \quad Z=t, \quad X=\sum_{k \geq 3} \alpha_{k} t^{k}, \quad Y=\sum_{k \geq 5} \beta_{k} t^{k}
$$

of $C$ at $P=(0,0,0,1)(=(X, Y, Z, W))$. We study the 2-minors of:

$$
\left[\begin{array}{ccc}
j W_{1}-i & \sum_{k \geq 3} \alpha_{k} W_{k} & \sum_{k \geq 5} \beta_{k} W_{k} \\
j & \sum_{k \geq 3} \alpha_{k} W_{k-1} & \sum_{k \geq 5} \beta_{k} W_{k-1}
\end{array}\right]
$$

We observe that modulo $\left(s_{1}, s_{2}\right)$ the first column is $\left(\begin{array}{c}-i \\ j\end{array}\right)$, which is non-zero for all $(i, j) \in$ $\mathbb{P}^{1}$. Hence our ideal is generated by the minors from the first and second, and first and third column. We then get the following generators:

$$
\begin{array}{r}
i \alpha_{3} s_{2}-i \alpha_{3} s_{1}^{2}+\left(2 i \alpha_{4}+j \alpha_{3}\right) s_{1} s_{2}-\left(i \alpha_{5}+j \alpha_{4}\right) s_{2}^{2} \text { modulo }\left(s_{1}, s_{2}\right)^{3}, \\
i \beta_{5} s_{2}^{2}-3 i \beta_{5} s_{1}^{2} s_{2}-3 i \beta_{6} s_{1} s_{2}^{2}-\left(i \beta_{7}+j \beta_{6}\right) s_{2}^{3} \text { modulo }\left(s_{1}, s_{2}\right)^{4} .
\end{array}
$$

If $\alpha_{3} \neq 0$, we insert $s_{2} \equiv s_{1}^{2}$ modulo $\underline{m}^{3}$, obtained from the first generator, in the second generator, which is then zero modulo $s_{1}^{4}$. If $\alpha_{3}=0$, we get a complete intersection of 
2 algebroid curves of degrees at least 2 . This gives multiplicity at least 4 , whatever the value of $\alpha_{3}$ is. This finishes the proof of Case 17).

Cases 8) AND 13). There is almost nothing to say about these 2 cases. One uses Lemmas 3.1 and 3.2 in Cases 8) and 13), respectively, and finds that $S_{R}$ has multiplicity at least 3 at $D$, for all $R \in L(D)$, and least 4 for those $R$ described in Theorem 2.2.d2) and f2), respectively. So we are in Cases (vi) and (vii), respectively.

R e mark. See Result 5.8 of [LP-II] which in these cases identifies the points $R$ such that $S_{R}$ has multiplicity at least 4 with points where $L(D)$ is tangent to some non-linear component $D\left(S_{i}\right)$ (or with vertices of cones of stationary bisecants). So these points are not necessarily cusps of some $D\left(S_{i}\right)$, but points where 2 components of $\mathcal{C}$ meet, and are tangent to each other.

CAsEs 9) AND 14). We will show that we are in Case (iv). It is clear that in Case 9) $L(D)$ is not a bitangent and satisfies neither $\mathrm{d} 1$ ) nor e1) of Theorem 2.2, but satisfies d) of Theorem 2.1 .

It is also clear that in Case 14) $L(D)$ satisfies f) of Theorem 2.1, but neither c1) nor f1) of Theorem 2.2. From straightforward application of Lemmas 3.1 and 3.2 it follows that the multiplicity of $S_{R}$ at $D$ is exactly 3 for all $R \in L(D) \backslash\{P, Q\}$ in Case 9), and all $R \in L(D) \backslash\{P\}$ in Case 14). The fact that $M(D)=\{Q\}$ in Case 9) and $M(D)=\{P\}$ in Case 14), follows directly from the discussion on pp. 219-220 of [LP-II].

CAsEs 4) AND 12). It is clear that $L(D)$ neither is bitangent at $P$ and $Q$ nor satisfies d) or e) of Theorem 2.1 in Case 4 ). It is clear that $L(D)$ satisfies neither c) nor f) of Theorem 2.1 in Case 12).

Furthermore it follows from direct calculations by using Lemmas 3.1 and 3.2 respectively, that $S_{R}$ has multiplicity exactly 2 for all $R \in L(D) \backslash\{P, Q\}$ and all $R \in L(D) \backslash\{P\}$ in Cases 4) and 12), respectively. It follows directly from the discussion on pp. 219-220 that $M(D)=\{Q\}$ in Case 4), and $M(D)=\{P\}$ in Case 12).

The cases left are 5),6), 11), and 16). In Case 5) it is clear that $L(D)$ satisfies e) of Theorem 2.1, and that $L(D)$ neither is bitangent to $C$ at $P$ and $Q$ nor satisfies $\mathrm{d} 1$ ) or e1) of Theorem 2.2. In Case 6) it is clear that $L(D)$ neither is bitangent nor satisfies d) or e) of Theorem 2.1. In Case 16) it is clear that $L(D)$ satisfies c) of Theorem 2.1, but neither c1) nor f1) of Theorem 2.2.

From straightforward calculations, by using Lemma 3.1, it follows that the multiplicity of $S_{R}$ at $D$ is exactly 3 for $R \in L(D) \backslash\{P, Q\}$ in Case 5), and exactly 2 for $R \in$ $L(D) \backslash\{P, Q\}$ in Case 6). Equally straightforward calculations, with the use of Lemma 3.2 , yield that the multiplicity of $S_{R}$ at $D$ is exactly 3 for $R \in L(D) \backslash\{P\}$ in Case 16).

What remains, is to prove the statements: $M(D)=\{P\}$ in Case 5) and 16), and $M(D)=\{Q\}$ in Case 6), and to carry out a proof in Case 11).

So far we have used two techniques to identify points of $M(D)$ for given $D$, namely the technique described in [LP-II], $\S 5$, and the local study of certain schemes performed in $[\mathrm{MS}]$. The method of $[\mathrm{LP}-\mathrm{II}], \S 5$, requires that $L(D)$ is tangent neither at $P$ nor at $Q$ in the case $D=P+Q$, and that $L(D)$ is not a flex in the case $D=P+Q$.

The method from [MS], used in order to study the curve $B(C)$ in Cases 1) and 2), is really developed to study a curve in $\check{\mathbb{P}}^{3}$, which is the union of the curves $B(C)$ and $D(C)$ (the strict dual of $C$, parametrizing osculating planes). This curve is locally isomorphic to $B(C)$ if we are outside $D(C)$, but not at points of $B(C) \cap D(C)$. In Cases 5$), 6), 11$ ) 
and 16) we are at points of $B(C) \cap D(C)$. Hence we cannot apply Theorem 2.2 of [MS] in these cases, as we could in Cases 1) and 2). Likewise, the methods of [LP-II], §5, cannot be used in Cases 5), 6), 16). In Case 11) the methods from [LP-II], $\S 5$, can be used to show the statement: $R \in M(D) \Leftrightarrow S_{R}$ has multiplicity at least 3 at $D$. (This is essentially Result 5.8 of [LP-II].) There is however no way to use these methods to show directly: " $R$ is a cusp of the branch of the $D\left(S_{i}\right)$ in question $\Leftrightarrow S_{R}$ has multiplicity at least 4 at $D$ ". In order to prove this one must find out when the osculating plane of $B(C)$ at $h$ is hyperosculating. We remark that in Case 11) $B(C)$ is smooth at $h$.

In Section 4 we will sketch a method for studying the curve $B(C)$ locally, Thereby completing the proof of our main results in Cases 5),6), 11), 16).

4. A local study of schemes parametrizing hyperplanes with prescribed intersection properties with respect to a fixed curve. Let $C$ be an abstract nonsingular curve over an algebraically closed field of characteristic zero. Let $V$ be a linear system on $C$, where the degree of $V$ is $d$, and the (affine) rank of $V$ is $r+1$. If $C$ is mapped into $\mathbb{P}^{r}$ by $V$, we think of $\mathbb{P} V$ as parametrizing the hyperplanes in $\mathbb{P}^{r}$, or as $\check{\mathbb{P}}^{r}$. For notational simplicity we will assume that $V$ is base-point free, and that $C$ is mapped into $\mathbb{P}^{r}$ as described. We then get an injective map $\phi$ from $\mathbb{P} V$ to $C_{d}$, where $C_{d}$ as before denotes the $d$-th symmetric product of (the abstract curve) $C$ with itself:

$$
\phi: \text { Hyperplane } H \longrightarrow \text { Divisor } H \cap C \text {. }
$$

Let $F \subseteq \mathbb{P} V \times C_{d}$ be the incidence correspondence

$$
\{(h, D) \mid D=\phi(h)\} .
$$

Let $X_{0}, \ldots, X_{r}$ be coordinates of $\mathbb{P}^{r}$, and assume that

$$
\phi(h)=d_{1} P_{1}+\ldots+d_{k} P_{k}, \text { where } \sum_{i=1}^{k} d_{i}=d .
$$

Let

$$
X_{i}=\sum_{\ell=0}^{\infty} \alpha_{i, j, \ell} t_{j}^{\ell}, \quad i=0, \ldots, r, \quad j=1, \ldots, k
$$

be local parametrizations of the embedded curve at $P_{1}, \ldots, P_{k}$. (If all $P_{i}$ are contained in the affine space $X_{r} \neq 0$, we choose $X_{r} \equiv 1$ in all these parametrizations.) Let $h$ be the point of $\mathbb{P} V$ corresponding to $X_{0}=0$. Let

$$
X_{0}+b_{1} X_{1}+\ldots+b_{r} X_{r}=0
$$

be the equation of "the general hyperplane" in an affine neighborhood Spec $k\left[b_{1}, \ldots, b_{r}\right]$ of $h$ (with $h$ at the origin).

If, for given $j$ in the range $1, \ldots, k$, we insert for each $X_{i}$ in (4.2) its local parametrization at $P_{j}$ given as (4.1), we obtain the expression:

$$
\sum_{\ell=0}^{d_{j}-1} A_{j, \ell}\left(b_{1}, \ldots, b_{r}\right) \cdot t_{j}^{\ell}+\sum_{\ell=d_{j}}^{\infty}\left(A_{j, \ell}\left(b_{1}, \ldots, b_{r}\right)+a_{j, \ell}\right) \cdot t_{j}^{\ell}
$$

where the $a_{j, \ell}$ are the $a_{0, j, \ell}$ above, and the $A_{j, \ell}$ are homogeneous linear combinations of the $b_{m}, m=1, \ldots, r$, depending on the $a_{i, j, \ell}$ for $i=1, \ldots, k, \ell=0, \ldots, \infty$. If $\phi(h)=$ 
$D=P_{1}+\ldots+P_{d}$, for $d=k$ distinct points, then it is obvious that the completion of the local ring of the incidence correspondence $F$ at $(h, \phi(h))$ is

$$
k\left[\left[b_{1}, \ldots, b_{r}, t_{1}, \ldots, t_{d}\right]\right] / \mathcal{F}
$$

where the ideal $\mathcal{F}$ is generated by the expressions $(4.3)$ for $j=1, \ldots, k=d$ (and $d_{j}=1$ for each $j$ ). Here we have identified $C_{d}$ with the Cartesian product $C^{d}$ locally at $D$. At the other extreme, it follows from [LP-I] that if $\phi(h)=D=d P$ (and we drop the index $j)$, then the completion of the local ring of $F$ at $(h, \phi(h))$ is:

$$
k\left[\left[b_{1}, \ldots, b_{r}, s_{1}, \ldots, s_{d}\right]\right] / \mathcal{F}
$$

where $s_{1}, \ldots, s_{d}$ (as well as $b_{1}, \ldots, b_{r}$ ) are treated as algebraically independent variables, and $\mathcal{F}$ is generated by:

$$
\sum_{\ell=0}^{d-1} A_{\ell}\left(b_{1}, \ldots, b_{r}\right) W_{\ell-s}+\sum_{\ell=d}^{\infty}\left(A_{\ell}\left(b_{1}, \ldots, b_{r}\right)+a_{\ell}\right) W_{\ell-s}
$$

for $s=0, \ldots, d-1$, and the $W_{i}$ are defined as:

$$
W_{i}=\left|\begin{array}{ccccc}
s_{1} & s_{2} & \ldots & \ldots & s_{i} \\
1 & s_{1} & \ldots & \ldots & s_{i-1} \\
0 & 1 & \ldots & \ldots & s_{i-2} \\
\vdots & \vdots & \ddots & \ddots & \vdots \\
0 & \ldots & 0 & 1 & s_{1}
\end{array}\right|, \quad i \geq 1
$$

$W_{0}=1, W_{i}=0$ for $i<0$, and $s_{j}=0$ for $j>d$. As an example, when $D=4 P$, we get:

$$
\begin{gathered}
A_{0}+A_{1} \cdot s_{1}+A_{2}\left(s_{1}^{2}-s_{2}\right)+A_{3}\left(s_{1}^{3}-2 s_{1} s_{2}+s_{3}\right) \\
+\left(A_{4}+\alpha_{4}\right)\left(s_{1}^{4}-3 s_{1}^{2} s_{2}+2 s_{1} s_{3}+s_{2}^{2}-s_{4}\right)+\ldots, \\
A_{1}+A_{2} \cdot s_{1}+A_{3}\left(s_{1}^{2}-s_{2}\right)+\left(A_{4}+\alpha_{4}\right)\left(s_{1}^{3}-2 s_{1} s_{2}+s_{3}\right)+\ldots, \\
A_{2}+A_{3} \cdot s_{1}+\left(A_{4}+\alpha_{4}\right)\left(s_{1}^{2}-s_{2}\right)+\ldots, \text { and } \\
A_{3}+\left(A_{4}+\alpha_{4}\right) s_{1}+\left(A_{5}+\alpha_{5}\right)\left(s_{1}^{2}-s_{2}\right)+\ldots
\end{gathered}
$$

as generators of $\mathcal{F}$, where $A_{i}=A_{i}\left(b_{1}, \ldots, b_{r}\right)$, for all $i$. These $d$ generators enable us to express $s_{1}, \ldots, s_{d}$ as explicit power series in the $A_{i}$ modulo the ideal $\mathcal{F}$. Starting from the top and beginning with calculation modulo $\left(b_{1}, \ldots, b_{r}\right)^{2}$, one gets

$$
\begin{aligned}
s_{d}= & (-1)^{d} \frac{A_{0}}{\alpha_{d}} \\
s_{d-1}= & (-1)^{d-1}\left[\frac{A_{1}}{\alpha_{d}}-\frac{\alpha_{d+1}}{\alpha_{d}^{2}} A_{0}\right] \\
s_{d-2}= & (-1)^{d-2}\left[\frac{A_{2}}{\alpha_{d}}-\frac{\alpha_{d+1}}{\alpha_{d}^{2}} A_{1}+\frac{\alpha_{d+2}^{2}-\alpha_{d+2} \alpha_{d}}{\alpha_{d}^{3}} A_{0}\right] \\
& \ldots \ldots \ldots] \\
s_{1}= & -\left[\frac{A_{d-1}}{\alpha_{d}}+\ldots\right]
\end{aligned}
$$

Then one starts from the top and calculates modulo $\left(b_{1}, \ldots, b_{r}\right)^{3}$ until one has calculated all $s_{i}$ modulo this power of $\left(b_{1}, \ldots, b_{r}\right)$. Calculating modulo higher and higher powers of 
$\left(b_{1}, \ldots, b_{r}\right)$ successively, one obtains expressions

$$
\begin{array}{cc}
s_{1}=f_{1}\left(b_{1}, \ldots, b_{r}\right) \\
\vdots \\
s_{d}=f_{d}\left(b_{1}, \ldots, b_{r}\right)
\end{array}
$$

If $D=d_{1} P_{1}+\ldots+d_{k} P_{k}$, we use the local, analytical isomorphism $\chi: C_{d} \simeq C_{d_{1}} \times \ldots \times C_{d_{k}}$ at $D$, and get $k$ sets of equations as above, expressing each element of $k$ sets of elementary symmetric functions as power series in $b_{1}, \ldots, b_{r}$ :

$$
\begin{array}{cccc}
s_{1,1}= & f_{1,1}(\underline{b}) & \ldots & s_{k, 1}=f_{k, 1}(\underline{b}) \\
\vdots & & & \vdots \\
s_{1, d_{1}}=f_{1, d_{1}}(\underline{b}) & \ldots & s_{k, d_{k}}=f_{k, d_{k}}(\underline{b})
\end{array}
$$

We now have the following

RESUlT 4.1. Let $\mathcal{M}$ be a subscheme of $C_{d}$, and let the completion of the local ring of $M$ at $D=\phi(h)$ be given via $\chi$ as

$$
k\left[\left[s_{i, j}\right]\right] / \mathcal{I}
$$

where $\mathcal{I}$ is generated by power series of the type

$$
g\left(s_{1,1}, \ldots, s_{k, d_{k}}\right) .
$$

Then the completion of the local ring of the inverse image scheme $\phi^{-1}(\mathcal{M})$ is:

$$
k\left[\left[b_{1}, \ldots, b_{r}\right]\right] / \mathcal{I}_{\phi},
$$

where $\mathcal{I}_{\phi}$ is generated by a set of power series

$$
g\left(f_{1,1}(\underline{b}) \ldots, f_{k, d_{k}}(\underline{b})\right)
$$

corresponding to the $g\left(s_{1,1}, \ldots, s_{k, d_{k}}\right)$ in a set of generators for $\mathcal{I}$.

This reduces the task of finding the completion of the local ring of a scheme of the type $\phi^{-1}(\mathcal{M})$, for $\mathcal{M} \subseteq C_{d}$, at a point $h$, to two problems

1) Finding suitable generators $g$ for $\mathcal{M}$ locally at $\phi(D)$.

2) Finding the expressions $f_{i, j}(\underline{b})$.

From now on we will use the same notation as in Section 3.

We now have developed most of the machinery needed to treat Case 11) and what is left of the proof of Cases 5), 6), 16).

CAse 11$) . D=2 P, L(D)$ is not a flex, and $I(P, C \cap H(D))=4$. Denote $H(D)$ by $h$. Since we are only interested in the branch of $\mathcal{S}^{\prime} \subseteq C_{2}$ corresponding to $D$, we can assume $\phi(h)=4 P+P_{1}+\ldots+P_{d-4}$, where the $P_{i}$ are distinct points (different from $P$ ). For all local purposes we can even assume that $d=4$, and $\phi(h)=4 P=2 D$. The curve $B(C)$ is then obtained as $\phi^{-1}(\mathcal{F})$. where $\mathcal{F}$ is the surface $\left\{2 E \mid E \in C_{2}\right\} \subseteq C_{4}$. The completion of the local ring of $\mathcal{F}$ at $4 P$ is

$$
k\left[\left[s_{1}, s_{2}, s_{3}, s_{4}\right]\right] / \mathcal{I},
$$

where $\mathcal{I}=\left(s_{3}-\frac{1}{2} s_{1} s_{2}+\frac{1}{8} s_{1}^{3}, s_{4}+\frac{1}{4} s_{1} s_{3}-\frac{1}{4} s_{2}^{2}+\frac{1}{64} s_{1}^{4}\right)$. Finding the first terms of the power series, $f_{1}, f_{2}, f_{3}, f_{4}$ (the pullbacks of $s_{1}, s_{2}, s_{3}, s_{4}$ via $\phi$, respectively), we see that 
modulo $\left(b_{1}, b_{2}, b_{3}\right)^{3}$, the pullbacks of the generators of $\mathcal{I}$ are:

$$
\begin{gathered}
\frac{A_{0}}{\alpha_{4}}-\frac{1}{4} \cdot \frac{A_{2}^{2}}{\alpha_{4}^{2}} \\
\text { and }-\frac{A_{1}}{\alpha_{4}}+\frac{\alpha_{5}}{\alpha_{4}^{2}} A_{0}+\frac{1}{2}\left[\frac{A_{3}}{\alpha_{4}}-\frac{\alpha_{5}}{\alpha_{4}^{2}} A_{2}\right]\left[\frac{A_{2}}{\alpha_{4}}\right] .
\end{gathered}
$$

We choose the parametrization

$$
\begin{array}{rrrrr}
W & =1 & & \\
Z & = & t & \\
X & = & & \\
Y & = & & \beta_{2} t^{2}+\beta_{3} t^{3}+\ldots \\
Y & & \alpha_{4} t^{4}+\alpha_{5} t^{5}+\ldots
\end{array}
$$

and take $Y+b_{1} X+b_{2} Z+b_{3} W$ as the equation of the general plane. Hence $A_{0}=b_{3}, A_{1}=$ $b_{2}, A_{\ell}=\beta_{\ell} b_{1}$, for $\ell \geq 2$.

We then get that modulo $\left(b_{1}, b_{2}, b_{3}\right)^{3}$ and modulo the pullbacks of the generators of $\mathcal{I}$, we have:

$$
\begin{aligned}
b_{3} & =\frac{1}{4} \frac{\beta_{2}}{\alpha_{4}^{2}} \cdot \beta_{2} \alpha_{4} \cdot b_{1}^{2} \\
b_{2} & =\frac{1}{4} \frac{\beta_{2}}{\alpha_{4}^{2}}\left(2 \beta_{3} \alpha_{4}-\beta_{1} \alpha_{5}\right) b_{1}^{2} .
\end{aligned}
$$

This implies that (the image of) $b_{1}$ is a parameter for the completion of the local ring of $B(C)$ at $h$, and that

$$
i_{0} b_{2}+j_{0} b_{3}=0
$$

is the equation of the osculating plane of $B(C)$ at $h$, where $\left(i_{0}, j_{0}\right)=\left(\beta_{2} \alpha_{4}, \beta_{2} \alpha_{5}-2 \beta_{3} \alpha_{4}\right)$. Hence $M(D)=R_{1}$, where $R_{1}=\left(0,0, \beta_{2} \alpha_{4}, \beta_{2} \alpha_{5}-2 \beta_{3} \alpha_{4}\right)$. Continuing with the same kind of calculation, this time modulo $\left(b_{1}, b_{2}, b_{3}\right)^{4}$, one finds that the osculating plane is hyperosculating if and only if

$$
\begin{aligned}
-\alpha_{4}^{2} \alpha_{6} \beta_{2}^{2} \beta_{3}+2 \alpha_{4} \alpha_{5} \alpha_{6} \beta_{2}^{3}-\alpha_{4}^{3} \beta_{3}^{3}+\alpha_{4}^{2} \alpha_{5} \beta_{2} \beta_{3}^{2}-2 \alpha_{4}^{2} \alpha_{5} \beta_{2}^{2} \beta_{4} \\
-\alpha_{4}^{2} \alpha_{7} \beta_{2}^{3}+\alpha_{4} \alpha_{5}^{2} \beta_{2}^{2} \beta_{3}-\alpha_{5}^{3} \beta_{2}^{3}+2 \alpha_{4}^{3} \beta_{2}^{2} \beta_{5}=0 .
\end{aligned}
$$

On the other hand, if $R=(X, Y, Z, W)=(0,0, i, j)$, then in order to find the intersection multiplicity of $S_{R}$ at $D$, we study the ideal generated by 2-minors of the matrix

$$
\left[\begin{array}{ccc}
j W_{1}-i & \beta_{2} W_{2}+\beta_{3} W_{3}+\ldots & \alpha_{4} W_{4}+\alpha_{5} W_{5}+\ldots \\
j & \beta_{2} W_{1}+\beta_{3} W_{2}+\ldots & \alpha_{4} W_{3}+\alpha_{5} W_{4}+\ldots
\end{array}\right]
$$

where

$$
W_{\ell}=\sum_{i=0}^{[\ell / 2]} s_{1}^{\ell-2 i} \cdot s_{2}^{i}, \text { for } \ell \geq 1 .
$$

One finds that the colenght of this ideal is at least 3 if and only if $(i, j)=\left(i_{0}, j_{0}\right)=$ $\left(\beta_{2} \alpha_{4}, \beta_{2} \alpha_{5}-2 \beta_{3} \alpha_{4}\right)$. Also one finds that for $(i, j)=\left(i_{0}, j_{0}\right)$ the colength is at least 4 if and only if a certain condition in $\alpha_{4}, \alpha_{5}, \alpha_{6}, \alpha_{7}, \beta_{2}, \beta_{3}, \beta_{4}, \beta_{5}$ holds. This condition turns out to be exactly the same as the condition that the osculating plane of $B(C)$ at $h$ is hyperosculating, in other words, that $R_{1}$ is a cusp of $D\left(S_{i}\right)$, for some branch $S_{i}$ of $B(C)$, or that $R_{1}$ is a vertex of a cone of bisecants.

Hence the proof is complete in Case 11). 
CASE 16). This case is similar to Case 11). In fact we get the same equations:

$$
\begin{aligned}
& \frac{A_{0}}{\alpha_{4}}-\frac{1}{4} \frac{A_{2}^{2}}{\alpha_{4}^{2}}=0 \\
&-\frac{A_{1}}{\alpha_{4}}+\frac{\alpha_{5}}{\alpha_{4}^{2}} A_{0}+\frac{1}{2}\left[\frac{A_{3}}{\alpha_{4}}-\frac{\alpha_{5}}{\alpha_{4}^{2}} A_{2}\right] \frac{A_{2}}{\alpha_{4}}=0 \\
& \text { modulo }\left(b_{1}, b_{2}, b_{3}\right)^{3} .
\end{aligned}
$$

Since $\beta_{2}=0$, we get $A_{2}=0$, hence the 2 equations reduce to:

$$
b_{2}=b_{3}=0 \quad \text { modulo }\left(b_{1}, b_{2}, b_{3}\right)^{3}
$$

in the completion of the local ring of $B(C)$ at $h$. This implies that $B(C)$ has a flex at $h$, and that we must check which linear combination

$$
i b_{2}+j b_{3} \text { that is of order at least } 4
$$

in the parameter $b_{1}$ in our ring.

Modulo $\left(b_{1}, b_{2}, b_{3}\right)^{4}$ our equations reduce to

$$
\begin{aligned}
& b_{3}=0 \\
& b_{2}=\frac{1}{8}\left[-\frac{\beta_{3} b_{1}}{\alpha_{4}}\right]^{3}
\end{aligned}
$$

Hence we see that $b_{3}=0$ is the equation of the osculating plane, corresponding dually to point $(0,0,0,1)$, which is $P$. Hence $M(D)=\{P\}$, as desired, and the proof is complete in Case 16).

Cases 5) And 6). We must show that $M(D)$ is $\{P\}$ and $\{Q\}$ in Cases 5) and 6), respectively. As in Cases 11) and 16) we study the scheme $\phi^{-1}(\mathcal{F})$, where $\phi: D \rightarrow L(D)$ is the natural map, and $\mathcal{F} \subseteq C_{d}$ consists of divisors of type $D^{\prime}+2 D^{\prime \prime}$, where $D^{\prime} \in C_{d-4}$, and $D^{\prime \prime} \in C_{2}$. As usual, let $h \in B(C)$ represent $H(D)$. Then $\phi(h)=3 P+r Q+E$, where $r=2$ in Case 6) and $r \geq 2$ in Case 5), and $P$ and $Q$ are outside the support of $E$. The branch of $B(C)=\phi^{-1}(\overline{\mathcal{F}})$ in question is the pull-back of 2 equations:

$$
\begin{array}{r}
27 s_{1,3}^{2}+4 s_{1,2}^{3}-18 s_{1,1} s_{1,2} s_{1,3}-s_{1,1}^{2} s_{1,2}^{2}+4 s_{1,1}^{3} s_{1,3}=0 \\
r^{r} s_{2, r}^{r-1}+(1-r)^{r-1} s_{2, r-1}^{r}+h_{r+1}+s_{2, r} h_{r-1}=0,
\end{array}
$$

where $h_{r \pm 1}$ consists of terms of degree $r \pm 1$ or more. Here $s_{1,1}, s_{1,2}, s_{1,3}$ are local parameters of $C_{3}$ at $3 P$, and $s_{2,1}, s_{2,2}, \ldots, s_{2, r}$ are local parameters of $C_{r}$ at $r Q$, and we work via the local analytical isomorphism

$$
C_{d} \simeq C_{3} \times C_{r} \times \ldots
$$

The rest of the proof in these cases consists of calculations that are contained in $[\mathrm{Pr}]$.

Remark 4.2. We also could have treated Cases 1) and 2) using the techniques developed in Section 4. Essentially we could have "reproduced" the power series given in Theorem 2.2 of [MS].

5. Some remarks. In this paragraph we will give some comments in connection with Theorems 2.1 and 2.2. We will use the words "expected" and "general" without always specifying what we mean by these terms. 
Remark 5.1. A general curve of degree $d$ and genus $g$ (whatever that is) is not "expected" to have any bitangent lines, flex lines, or planes intersecting the curve more than four times at a point. Moreover, for each tangential trisecant (reintersecting tangent) line, the osculating plane at the point of (line) tangency is not expected to be tangent to $C$ at the point of (line) reintersection. Hence it follows from Theorems 2.1 and 2.2 that for "a general curve of degree $d$ and genus $g$ " we "expect":

$$
\left\{R \in \mathbb{P}^{3} \mid a_{1} \geq 3\right\}=\bigcup_{i} D\left(S_{i}\right) \cup\{\text { vertices of cones of bisecant to } C\},
$$

and the closure of $\left\{R \in \mathbb{P}^{3} \mid a_{1}=3\right\}=\bigcup_{i} D\left(S_{i}\right)$, where $\left\{S_{i}\right\}=$ non-degenerate components of $B(C)\}$. It is not clear to us whether one should expect $B(C)$ to be irreducible for general $C$ of high enough degree and specified genus. For $d=4, g=1$ we have seen that $B(C)$ consists of four plane conic components, moreover $\left\{R \in \mathbb{P}^{3} \mid a_{1} \geq 3\right\}=\{R \in$ $\left.\mathbb{P}^{3} \mid a_{1}=\infty\right\}=C \cup\{4$ points $\}$ in this case (for a general intersection of 2 quadrics). We sum up by:

In rough terms, it follows from Theorem 2.1 and 2.2 that in "most cases" the closure of $\left\{R \in \mathbb{P}^{3} \mid a_{1}=3\right\}=D(B(C))=$ cuspidal edge of the surface $F_{3}$ of Section 1 .

We could also add:

From Theorem 2.2 one may deduce that in "most cases" $\left\{R \in \mathbb{P}^{3} \mid a_{1} \geq 4\right\}=$ \{cusps of $D(B(C))\} \cup C=\left\{\right.$ cusps of cuspidal edge of the $\left.F_{3}\right\} \cup C$.

Remark 5.2. Table of "expected" values of a curve $C$ of degree $d$ and genus $g$. See also [vzG], p. 607.

\begin{tabular}{|c|c|c|c|c|}
\hline Curve & Degree & $\begin{array}{c}\text { Genus } \\
\text { (geometric) }\end{array}$ & \# Cusps & $\begin{array}{c}\text { \# Cusps } \\
\text { outside } C\end{array}$ \\
\hline$C$ & $d$ & $g$ & 0 & 0 \\
\hline$C_{G}$ & $2 d+2 g-2$ & $g$ & 0 & - \\
\hline$D(C)$ & $3 d+6 g-6$ & $g$ & $4 d+12 g-12$ & - \\
\hline$D(B(C))$ & $6 d^{2}+12 d g$ & $2 d^{2}+6 d g$ & $14 d^{2}+34 d g$ & $12 d^{2}+32 d g+20 g^{2}$ \\
& $+6 g^{2}-36 d$ & $+4 g^{2}-15 d$ & $+20 g^{2}-94 d$ & \\
& $-60 g+54$ & $-31 g+28$ & $-176 g+156$ & $-84 d-164 g+144$ \\
\hline $\mathcal{S}$ & $2 d^{2}+2 d g-8 d$ & $2 d^{2}+6 d g$ & 0 & - \\
& $-6 g+6$ & $+4 g^{2}-15 d$ & & \\
& & $-31 g+28$ & & - \\
\hline$B(C)$ & $2 d^{2}+4 d g$ & $2 d^{2}+6 d g$ & $6 d^{2}+18 d g$ & \\
& $+2 g^{2}-10 d$ & $+4 g^{2}-15 d$ & $+12 g^{2}-42 d$ & \\
& $-14 g+12$ & $-31 g+28$ & $-84 g+72$ & \\
\hline
\end{tabular}

Here $C_{G}$ is the curve in $G(1,3)$ parametrizing tangent lines, and we recall that $\mathcal{S} \subseteq G(1,3)$ parametrizes stationary bisecant lines to $C$. The values in the table are calculated under the assumption that $B(C), \mathcal{S}^{\prime}, \mathcal{S}$ and $D(B(C))$ are irreducible. The degree and number of cusps of $B(C)$ are determined by the De Jonquieres formula. See for example [ACGH], p. 359. The cusps of $B(C)$ correspond to osculating planes that are tangent to $C$ elsewhere. At these cusps $B(C)$ intersects $D(C)$, which is expected to be smooth at these points. Otherwise $B(C)$ intersects $D(C)$ at the points corresponding to hyperosculating planes of $C$, here $D(C)$ has cusps, while $B(C)$ is expected to be smooth. Elsewhere $B(C)$ is expected to have a finite number (see $[\mathrm{ACGH}]$, p. 364) of trinodes, corresponding to 
tritangent planes of $C$ while $D(C)$ is expected to be smooth.

Furthermore it was shown in [LP-II], $\S 5$, that $\mathcal{S}^{\prime} \subseteq C_{2}$ is expected to be smooth. $\mathcal{S}^{\prime}$ is expected to differ from $\mathcal{S} \subseteq G(1,3)$ locally, only at points corresponding to tangential trisecant lines. From the study of Case 2) in Section 3 it follows that at such points $B(C)$ is expected to be smooth and have no flexes. Hence $\mathcal{S}$ will be smooth also at such points, and $\mathcal{S}$ is therefore expected to be smooth. The common expected geometric genus of $B(C), \mathcal{S}, \mathcal{S}^{\prime}$, and $D(B(C))$ is then determined by the correspondence

$$
\begin{aligned}
C & -\mathcal{S}^{\prime} \\
P & \longrightarrow\left\{\text { All } P+P^{\prime} \mid P+P^{\prime} \in \mathcal{S}^{\prime}\right\} \\
\left\{P, P^{\prime}\right\} & \longleftarrow D=P+P^{\prime} .
\end{aligned}
$$

A general $P$ corresponds to $2 d+2 g-6$ divisors. A general $D \in \mathcal{S}^{\prime}$ corresponds to two points. A more precise statement in the sense of $[\mathrm{GH}]$, p. 282 , is to say that the correspondence is given as a holomorphic map $f: C \rightarrow \mathcal{S}_{2 d+2 g-6}^{\prime}$, where $f(P)=\sum e_{i}(P+$ $P_{1}^{\prime}$ ), and $e_{i}$ is the multiplicity of $P_{i}^{\prime}$ as a Wronskian point of the (affine) rank 2 linear system $V(-2 P)$ on $C$. Each $P$ such that there is a tangential trisecant simply tangent to $C$ at $P$, and simply intersecting $C$ at $P_{1}^{\prime}$; and each $P$ such that an osculating (but not higher osculating) plane at another point $P_{1}^{\prime}$ simply touches $C$ at $P$, corresponds to only $2 d+2 g-7$ points of $\mathcal{S}^{\prime}$. In each of these cases $e_{1}=2$, arising from vanishing sequences $(1,2)$ and $(0,3)$ of $V(-2 P)$ at $P_{1}^{\prime}$ respectively (as opposed to $(0,1)$ for a general point of $C$ and $(0,2)$ for $P_{1}^{\prime}$ such that we have an "usual" bitangent plane touching at $P$ and $\left.P_{1}^{\prime}\right)$. Hence $\left(P, P+P_{1}^{\prime}\right)$ appears with multiplicity $e_{1}-1=1$ in the ramification divisor of the map from the graph curve of the correspondence down to $C$. A general curve is "expected" to have only such tangential trisecants and 3-2-planes as just described.

Each $D=2 P$, such that the osculating plane of $C$ at $P$ is hyperosculating, corresponds to only one point $P$ on $C$. The curve is expected to have $\delta_{1}=2 d^{2}+2 d g-10 d-12 g+12$ tangential trisecants, $\delta_{2}=6 d^{2}+18 d g+12 g^{2}-42 d-84 g+72$ osculating planes touching $C$ elsewhere, and $\delta^{\prime}=4 d+12 g-12$ hyperosculating planes. The correspondence gives that $p$, the genus of $\mathcal{S}^{\prime}$, is determined by

$$
\begin{array}{ll} 
& (2 d+2 g-6)(2 g-2)+\delta_{1}+\delta_{2}=2(2 p-2)+\delta^{\prime}, \\
\text { so } \quad & p=2 d^{2}+6 d g+4 g^{2}-15 d-31 g+28 .
\end{array}
$$

The rest of the numbers are determined by the standard Plücker formulas. The difference between the total number of cusps of $D(B(C))$ and the number of cusps outside $C$ is due to the $\delta_{1}$ tangential trisecants, which give rise to cusps of $D(B(C))$ at the points where these secant lines are tangent to $C$. The expected $\delta_{2}$ osculating planes to $C$ that touch $C$ elsewhere, give rise to $\delta_{2}$ additional points of $C \cap D(B(C))$. These are the points $Q$ of Case 4) of Section 3. At these points $C$ and $D(B(C))$ are expected to be smooth and intersect transversally.

We "expect" the following strata to non-empty for a curve $C$ with $\Delta(d, g)=\frac{1}{2}(d-$ 1) $(d-2)-g \geq 4$.

\section{Partition}

$1 \geq 1 \geq \ldots \geq 1 \geq 1$

$2 \geq 1 \geq \ldots \geq 1$

$3 \geq 1 \geq \ldots \geq 1$

\section{Closure of stratum}

$\mathbb{P}^{3}$

Surface $F_{3}$ of degree $2 d^{2}+2 d g-8 d-6 g+6$

Curve $D(B(C))$

of degree $6 d^{2}+12 d g+6 g^{2}-36 g-60 g+54$ 
$2 \geq 2 \geq 1 \ldots \geq 1$

$3 \geq 2 \geq 1 \ldots \geq 1$

$2 \geq 2 \geq 2 \geq 1 \geq \ldots \geq 1$

$4 \geq 1 \geq \ldots \geq 1$ and genus $2 d^{2}+6 d g+4 g^{2}-15 d-31 g+48$

The curve which is the closure of $B(B(C)) \backslash C$; of degree $2 d^{4}+4 d^{3} g+2 d^{2} g^{2}-18 d^{3}-32 d^{2} g$ $-14 d^{2} g+46 d^{2}+52 d g+8 g^{2}-6 d+64 g-72$

Finite stratum: $(D(B(C)) \cap B(B(C)) \backslash C$.

Points where a tangent line to $D(B(C))$ reintersects $D(B(C))$.

Finite stratum:

\{triple points on the nodal edge of $\left.F_{3}\right\} \backslash C$

Finite stratum: $12 d^{2}+32 d g+20 g^{2}$

$-84 d-164 g+144$ cusps of $D(B(C)) \backslash C$.

Remark 5.3. So far we have described a "projection-stratification" of $\mathbb{P}^{3}$ due to a curve $C$. Similarly a reduced curve $C \subseteq \mathbb{P}^{3}$ gives rise to a "section-stratification" of $\check{\mathbb{P}}^{3}$, We say that a point $h \in \check{\mathbb{P}}^{3}$, representing a plane $H \subseteq \mathbb{P}^{3}$, is contained in the section-stratum

$$
b_{1} \geq b_{2} \geq \ldots \geq b_{k}
$$

if $H \cap C=b_{1} P_{1}+b_{2} P_{2}+\ldots+b_{k} P_{k}$, for distinct points $P_{i}$.

Of course $b_{i}=I\left(P_{i}, C \cap H\right)$ for each $P_{i}$, and $\sum_{i=1}^{k} b_{i}=d=$ degree of $C$. Then we have:

$\left\{h \mid b_{1} \geq 2\right\}=$ dual surface of $C$, parametrizing planes that are tangent to $C$;

$\left\{h \mid b_{2} \geq 2\right\}=B(C)=$ nodal edge of the dual surface of $C$;

$\left\{h \mid b_{2} \geq 3\right\}=B(C)=$ strict dual curve of $C=$ cuspidal edge of the dual surface of $C=\{h \mid h$ corresponds to an osculating plane of $C\}$;

$\left\{h \mid b_{1} \geq 4\right\}=\{$ cusps of the strict dual curve of $C\}$ $=\{h \mid h$ corresponds to a hyperosculating plane of $C\}$.

One can say that Theorems 2.1 and 2.2 describe a certain relationship between the projection-stratification of $\mathbb{P}^{3}$ due to $C$, and the section-stratification of $\mathbb{P}^{3}$ due to $B(C) \subseteq$ $\check{\mathbb{P}}^{3}$, at least for curves, which "behave as expected."

R e mark 5.4. Ignacio Sols has asked a question, which in the language of our paper is as follows: "Let $G$ be monodromy (or Galois) group of the points of $S_{R}$ as $R$ varies over $\mathbb{P}^{3}$. For which integral smooth non-degenerate curves in $\mathbb{P}^{3}$ is the group $G$ the full symmetric group in $\Delta(d, g)=\frac{1}{2}(d-1)(d-2)-g$ letters?" For $d=3, g=0$, there is nothing to prove; for $d \geq 4$ the question is essentially: Is the stratum $\{2 \geq 1 \ldots \geq 1\}$ non-empty? It is clear that if $d \geq 4$ then $\left\{R \mid a_{1} \geq 2\right\}$ is a surface. What one has to prove, is that this surface is not the union of the sets $\left\{R \mid a_{1} \geq 3\right\}$ and $\left\{R \mid a_{2} \geq 2\right\}$. It is clear from Theorem 2.1 that all components of $\left\{R \mid a_{1} \geq 3\right\}$ have dimension at most one. One can not prove any such statement for the set $\left\{R \mid a_{2} \geq 2\right\}$.

Indeed, in $[\mathrm{BM}]$ one constructs a rational sextic curve $C$ with a one-dimensional family of stationary trisecants, that is: trisecants such that for each trisecant the tangent lines to $C$ at the points of secancy are in the same plane. In addition $C$ possesses 6 bitangent lines. In this case $B(C)$, which according to the table in 5.2 is expected to have degree 24 , breaks up into a non-linear component of degree 6 counted 3 times, and 6 lines, which are dual to the bitangent lines. The surface, which is the tangent developable of the strict dual curve of the non-linear component of $B(C)$, and at the same time the union of the stationary trisecant lines, has degree 10 . Since each stationary trisecant is a stationary 
bisecant in 3 ways, this corresponds well with the expected degree of $\mathcal{S}$, which is 30 . In $[\mathrm{BM}]$ one proves rigorously that any pair of coplanar tangents is a part of a triple of coplanar tangents, such that the 3 points of tangency are collinear. Hence $a_{2} \geq 2$ (in fact: $a_{3} \geq 2$ ), for each point $R$ on any such line. At each of the 12 points $P$ where the osculating plane is hyperosculating, the tangent line meets $C$ in another point $Q$, where the tangent line of $C$ at $Q$ is contained in the plane of hyper-osculation at $P$. Hence both $2 P$ and $P+Q$ appear with multiplicity at least 2 in $V_{R}$ for any point $R$ on the line $P Q$ outside $C$. For each point $R$ on any of the 6 bitangent lines we of course have $a_{1} \geq 4$.

Hence it is clear that the stratum $\{2 \geq 1 \geq 1 \ldots \geq 1\}$ is empty for the rational sextic described in $[\mathrm{BM}]$. It would be interesting to investigate whether this curve is the only one for $d \geq 4$ with an empty stratum $\{2 \geq 1 \geq 1 \ldots \geq 1\}$.

Another example of a curve $C$ with a surface stratum $\{2 \geq 2 \geq 2 \geq 1 \ldots \geq 1\}$ is a complete intersection of a quadric cone $\mathcal{T}$ and a cubic surface. For all points on $\mathcal{T}$ we have either $a_{2} \geq 2$, or $a_{1} \geq 3$. A general point on a generatrix $L$ of the cone intersets $C$ in 3 points $P_{1}, P_{2}, P_{3}$, giving rise to three points $P_{1}+P_{2}, P_{1}+P_{3}, P_{2}+P_{3}$ of $S_{R}$, each of multiplicity 2 , since the tangent plane of $\mathcal{T}$ is constant along each generatrix. $C$ has degree 6 and genus 4 , and the expected degree of the curves $T$ and $\mathcal{S}$ (parametrizing trisecants and stationary bisecants, respectively) in $G(1,3)$ are 4 and 54 , respectively. The conic component corresponding to lines on $\mathcal{T}$ in a natural way counts twice on $T$, and "fills up" $T$. This component only counts 3 (and not 27) times on $\mathcal{S}$, and therefore we expect other stationary bisecant lines in this case.

In the two examples of sextic curves $C$ just mentioned it is not possible to interpret the projection-stratum $\{2 \geq 2 \geq 2 \geq 1 \ldots \geq 1\}$ with respect to $C$ as a section stratum of $\mathbb{P}^{3}$ with respect to a reduced curve $B$. No curve has a 2-dimensional family of tritangent (not even bitangent) planes. On the other hand, any sensible scheme-theoretical description of $B(C)$ (as a subscheme of $\mathbb{P}^{3}$ ) for example the one in Section 4, will give a component of $B(C)$ that appears with multiplicity at least $\left(\begin{array}{l}3 \\ 2\end{array}\right)=3$, so any tangent plane to this component of $B(C)$; corresponding dually to a point of $F_{3}=\left\{R \mid a_{1} \geq 2\right\}$; is in a sense tritangent, in virtue of being a tangent plane at all.

Remark 5.5. If $d=3, g=0$ then $\Delta(d, g)=\frac{1}{2}(d-1)(d-2)-g=1$. Hence $\left\{R \mid a_{1} \geq 2\right.$ and $a_{1}$ finite $\}=\emptyset$.

If $d=4, g=1$, then $\Delta(d, g)=2$. Hence $\left\{R \mid a_{1} \geq 3\right.$ and $a_{1}$ finite $\}=\emptyset$. It follows from Theorem 2.1 that there cannot be any non-degenerate component of $B(C)$. Hence all components of $B(C)$ are plane, and the surface $\mathcal{S}$ is a union of cones. The expected degree 8 corresponds to 4 quadric cones. The expected genus -3 of $B(C)$ makes sense as the arithmetic genus of 4 disjoint conics. The expected degree 0 of $D(B(C))$ also makes sense, since there is no such curve. It is hard, however, to find natural explanation for the -16 expected cusps of the non-existent curve $D(B(C))$.

If $d=4, g=0$ then $\Delta(d, g)=3$. Hence $\left\{R \mid a_{1} \geq 4\right.$ and $a_{1}$ finite $\}=\emptyset$. It follows from Theorems 2.1 and 2.2 that there are no cusps of $D(B(C))$ outside \{vertices of cones of bisecants . From our table in Remark 5.3 we see that all 4 expected cusps of the rational curve $D(B(C))$ of degree 6 are points of $C$, since $C$ possesses 4 tangential trisecants. Hence we expect no cusps of $D(B(C))$ outside $C$.

If $d=5, g=2$ then $\Delta(d, g)=4$. Then we expect 96 cusps of $D(B(C))$ outside $C$. From the table in Remark 5.2 it follows that we always expect cusps of $D(B(C)$ ) when $\Delta(d, g) \geq 4$. 
Remark 5.6. A dense open set of the curve $C$ itself is expected to appear as a part of the section stratum

$$
2 \geq 2 \geq \ldots \geq 2 \geq 1 \geq \ldots \geq 1
$$

with respect to $B(C)$, where the number 2 occurs $2 d+2 g-6$ times in the partition. We recall that $2 d+2 g-6$ is the number of stationary bisecants through a general point on $C$. This implies that $C$ appears as a $\left(\begin{array}{c}2 d+2 g-6 \\ 2\end{array}\right)=\left(2 d^{2}+4 d g+2 g^{2}-13 d-13 g+21\right)$ fold component of the curve $B(B(C))$. Therefore the expected degree of the projectionstratum

$$
2 \geq 2 \geq 1 \ldots \geq 1
$$

with respect to $C$ is calculated in the following way:

$$
\frac{1}{2}(\operatorname{deg} \mathcal{S}-1)(\operatorname{deg} \mathcal{S}-2)-\operatorname{genus} \mathcal{S}-\operatorname{deg}(D(B(C)))-d \cdot\left(\begin{array}{c}
2 d+2 g-6 \\
2
\end{array}\right) .
$$

(Take a plane section of $\mathcal{S}$.) Clearly this number is zero for $\Delta(d, g)<4$, that is, for $d \leq 4$. In the case $d=4, g=0$, we see that $B(C)$ is a smooth rational quartic curve just like $C$, and that $B(B(C))=C$. Hence we have some sort of "auto-bitangent duality" in this case.

In the case $d=4, g=1$, we bravely claim that $B(B(C))=C \cup\{4$ points $\}$, and that $C$ in a natural way is a 6 -fold component of $B(B(C))$, since all points on $C$ correspond dually to planes that are simultaneously tangent to each of the 4 conics of $B(C)$.

\section{References}

[ACGH] E. Arbarello, M. Cornalba, P. A. Griffiths, and J. Harris, Geometry of algebraic curves, Vol. I. Springer Verlag, New York, 1985.

[BM] W. Barth, R. Moore, On rational plane sextics with six tritangents, in: Algebraic Geometry and Commutative Algebra, Vol. I, 45-58, Kinokuniya, Tokyo, 1988.

[Bo] J. M. Boardman, Singularities of Differentiable Maps, Inst. Hautes Études Sci. Publ. Math. 33 (1967), 21-57.

[vzG] J. von zur Gathen, Secant Spaces to Curves, Canad. J. Math. 35 (1983), 589-612.

[GH] P. Griffiths, J. D. Harris, Principles of Algebraic Geometry, Pure \& Applied Mathematics, Wiley, 1978.

[LP-I] M. E. Huibregtse, T. Johnsen, Local Properties of Secant Varieties in Symmetric Products, Part I. Trans. Amer. Math. Soc. 313 (1989), 187-204.

[LP-II] T. Johnsen, Local Properties of Secant Varieties in Symmetric Products, Part II. Trans. Amer. Math. Soc. 313 (1989), 205-220.

[MS] T. Johnsen, Multiplicities of Solutions to some Enumerative Contact Problems, Math. Scand. 63 (1988), 87-108.

[Pr] T. Johnsen, Plane projections of a smooth space curve, Preprint No. 8, Mathematics Reports, University of Tromsø, 1990.

[Ro] J. Roberts, Singularity Subschemes and Generic Projections, Trans. Amer. Math. Soc. 212 (1975), 229-268.

[Te] B. Teissier, The Hunting of Invariants in the Geometry of Discriminants, Proc. Nordic Summer School; Real and Complex Singularities, Oslo 1976, Ed. Per Holm, Sijthoff and Hoordhoff, 1977.

[Th] R. Thom, Les Singularités des Applications Différentiables, Ann. Inst. Fourier (Grenoble) 6 (1955/56), 43-87. 